Canadian

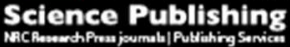

Canadian Journal of Zoology Revue canadienne de zoologie

\title{
Pattern of ovulation in an ancient, solitary mole-rat lineage: Heliophobius argenteocinereus emini from Tanzania
}

\begin{tabular}{|r|l|}
\hline Journal: & Canadian Journal of Zoology \\
\hline Manuscript ID & cjz-2016-0292.R1 \\
\hline Manuscript Type: & Article \\
\hline Complete List of Authors: & $\begin{array}{l}\text { Katandukila, Jestina; University of Dar es Salaam, zoology } \\
\text { Ngalameno, Mungo; University of Pretoria, Mammal Research Institute, } \\
\text { Department of Zoology \& Entomology; Sokoine University of } \\
\text { Agriculture, Anatomy } \\
\text { Mgode, Georgies; Sokoine University of Agriculture, Pest Management } \\
\text { Centre } \\
\text { Bastos, Armanda; Mammal Research Institute, Zoology and Entomology } \\
\text { Bennett, Nigel; Mammal Research Institute, }\end{array}$ \\
\hline Keyword: & $\begin{array}{l}\text { Heliophobius, progesterone, OVULATION < Organ System, penile spines, } \\
\text { coitus }\end{array}$ \\
\hline \multicolumn{2}{|c}{} \\
\hline
\end{tabular}

\section{SCHOLARONE \\ Manuscripts}




\title{
Pattern of ovulation in an ancient, solitary mole-rat lineage: Heliophobius argenteocinereus emini from Tanzania
}

\author{
Jestina V. Katandukila ${ }^{1,2}$, Mungo, K. Ngalameno ${ }^{1}$, Georgies, F. Mgode ${ }^{3}$, Armanda, D.S. \\ Bastos $^{4}$ and Nigel C. Bennett ${ }^{1}$ \\ ${ }^{1}$ South African Research Chair for Mammal Behavioural Ecology and Physiology, \\ Department of Zoology and Entomology, University of Pretoria, Pretoria 0002, South \\ Africa \\ ${ }^{2}$ Department of Zoology and Wildlife Conservation, College of Natural and Applied \\ Sciences, University of Dar es Salaam, P.O. Box 35064, Dar es Salaam, Tanzania \\ ${ }^{3}$ Pest Management Centre, Sokoine University, P.O. Box 3110, Morogoro, Tanzania \\ ${ }^{4}$ Mammal Research Institute, Department of Zoology and Entomology, University of \\ Pretoria, Pretoria 0002, South Africa
}

\begin{abstract}
Heliophobius is an ancient subterranean rodent lineage within the family Bathyergidae, that displays seasonal breeding over a broad geographical range. In East Africa, reproduction in these solitary mole-rats has been shown to coincide with the long rains, but it is not clear whether Heliophobius argenteocinereus emini (Noack 1894) from Tanzania is an induced or spontaneous ovulator. To address this, urinary progesterone was measured every second day over a period of 132 days in six wild-caught females subjected to three sequential experimental treatments. In the first, control (C) treatment, females were housed singly for a period of 44 days, following which non-physical contact (NPC) with a vasectomised male and then physical contact (PC) with the same vasectomised male, occurred, in each of the subsequent 44-day treatments. Non-invasive monitoring of ovarian cyclicity confirmed that
\end{abstract}


mean urinary progesterone concentration was higher during PC than during either NPC or C, despite the fact that the males were vasectomised and incapable of fertilising the females. Examination of penile morphology revealed that males possess epidermal projections on the glans penis which probably bring about cervical stimulation during coitus. These findings together with the female progesterone profiles imply the species is an induced ovulator stimulated by penile intromission.

Keywords: Heliophobius, progesterone, ovulation, penile spines, coitus

\section{INTRODUCTION}

The genus Heliophobius (Peters 1846) comprises solitary subterranean rodents belonging to the family Batherygidae. Among batherygids, the genus Heliophobius has a broad geographic distributional range in eastern and southern Africa including Tanzania, the Democratic Republic of Congo, Kenya, Malawi, Mozambique and Zambia (Faulkes et al. 2011). It represents the second oldest lineage within the African mole-rat family and occurs as a cryptic species complex within which at least six topotypical mitochondrial lineages are recognised (Faulkes et al. 2011). Heliophobius argenteocinereus emini is herbivorous, feeding on a variety of geophytes with underground storage organs and particulary fond of tubers in agricultural fields. They inhabit highland savannah and woodlands in areas characterised by mean annual rainfall $>900 \mathrm{~mm}$ (Škliba et al. 2007).

Heliophobius argenteocinereus emini is a seasonal breeder with reproduction closely linked to the latter part of the long rains (Šumbera et al. 2003; Ngalameno et al. 2017). Reproduction is restricted to a single breeding event per year with a litter size that ranges from 3-6 per adult female. Reproductive activity arises during the middle of the long rains with birth peaking at the end of the long rainy season (Šumbera et al. 2003a; 2008; Ngalameno et al. 2017). 
Rainfall is important for initiating the onset of reproductive activity in mole-rats since it promotes burrowing activity that enables the successful location of geophytes (Bennett et al. 2000) and dispersal of sub-adults prior to the annual breeding cycle as reported in other subterranean rodents (Jarvis and Sale 1971; Heth 1989; Šumbera et al. 2003b; Herbst et al. 2004; Herbst and Bennett 2006; Thomas et al. 2009; Katandukila et al. 2014).

Ovulation may be spontaneous or induced by the physical process of coitus and/or semiochemical (i.e. pheromones) stimuli (Conaway 1971; Feder 1981; Hickok and Tischkau 2010). Indeed, in mole-rats the presence of an unrelated male, but no physical contact may result in heightened progesterone concentrations (Faulkes et al. 2010; Bennett et al. 2010; Katandukila et al. 2016), these rises in pregesterone are due to the formation of luteinised unruptured follicles that arise from developing follicles which fail to ovulate because of a lack of coitus.

Spontaneous ovulation results from a continuous cycle of follicular development where primordial follicles develop into Graafian follicles, and following ovulation a corpus luteum arises. Spontaneous ovulation is under the control of the cyclical production of hormones (Milligan 1974). The naked mole-rat, (Heterocephalus glaber) (Ruppell 1842) the Damaraland mole-rat (Fukomys damarensis)(Ogilby 1838), the giant mole-rat, (Fukomys mechowii)(Peters 1881) and Ansell's mole-rat, (Fukomys anselli) (Burda, Zima, Scharff, Macholan and Kawalika 1999) are social (Faulkes et al. 1990; Snyman et al. 2006; Bennett et al. 2010; Faulkes et al. 2010) and all exhibit a spontaneous patterns of ovulation.

Induced ovulation, on the otherhand essentially arises as a result of cervical stimulation that promotes the release of gonadotropin-releasing hormone $(\mathrm{GnRH})$ from the hypothalamus which in turn brings about a surge of luteinising hormone (LH) with the consequence of ovulation. Semiochemical stimuli are important in mate receptivity and in conjunction with coitus is important for the surge of LH (Sellix and Menaker 2010). The tactile stimuli caused by the outer surface of penis during copulation against the vaginal wall and cervix is crucial 
for bringing about ovulation in induced ovulators (Jöchle 1975; Viker et al. 1993; Parag et al. 2006). Multiple attempts of mounting and copulation are needed to stimulate induced ovulation and the subsequent production of a corpus luteum of ovulation, if fertilization is to occur (Bennett et al. 2000). Induced ovulation has been recorded in a number of species of African mole-rats including the Cape dune mole-rat (Batherygus suillus (Schreber 1782); Hart et al. 2006), social Natal mole-rat (Cryptomys hottentotus natalensis (Roberts 1913); Jackson and Bennett 2005), social highveld mole-rats (Cryptomys hottentotus pretoriae (Roberts 1913); Malherbe et al. 2005) and Cape mole-rat (Georychus capensis (Pallas 1778); van Sandywk and Bennett 2005). Other solitary subterranean and fossorial rodents which exhibit induced ovulation include the blind mole-rat (Spalax ehrenbergi (Nehring 1898); Shanas et al. 1995), the Tuco tuco (Ctenomys talarum (Thomas 1898); Fanjul and Zenuto 2012) and the East African root rat (Ruppell 1835) (Tachyoryctes spendens; Katandukila and Bennett 2016). Currently nothing is known about the pattern of ovulation arising in the genus Heliophobius.

Induced ovulation has been demonstrated in solitary subterranean rodents from a several aspects; the profiles of progesterone hormone of females as well as the the investigation of the outer surface of the glans of the males (Parag et al. 2006; Faulkes et al. 2010; Katandukila and Bennett 2016). Induced ovulation reduces reproductive costs to ovulating. and increases the probability of successful breeding after copulation (Lariviere and Ferguson 2003). Solitary species as well as species with large home ranges or low population densities that have low mate encounters may benefit from this pattern of ovulation (Lariviere and Ferguson 2003).

In this study the primary aims were to a) measure the urinary progesterone concentrations in females with the hope of assessing the potential ovarian cycles; b) investigate the epidermal surface of the penis in captive male Heliophobius to ascertain if spinal projections are present on the glans and/ or shaft. 
We predicted that female Heliophobius argenteocinereus emini would exhibit ovarian cyclicity, but only when paired with an unrelated male conspecific and allowed copulation. We further predicted that females would only exhibit elevated progesterone concentrations resulting from the production of corpora lutea of ovulation following full phyical contact with a male that had been vasectomised. Lastly, we predicted that males would possess epidermal spines on the surface of the glans.

\section{MATERIALS AND METHODS}

A total of five male and six female Heliophobius a. emini were captured from farmlands in the Morogoro region of Tanzania (06 $57^{\circ} 16.45-48^{\prime \prime}$ S, 037 32'05.40-47"E; 1266-1330 metres above sea level) in January 2015. They were initially maintained at the Department of Zoology and Wildlife Conservation, University of Dar es Salaam, Tanzania for three weeks prior to subsequent exportation to the Department of Zoology and Entomology, University of Pretoria, Republic of South Africa. The mole-rats were housed singly. The mole-rats were fed on chopped sweet potato, apples and occasionally grapes and carrots, they were maintained within the laboratory at $25^{\circ} \mathrm{C}$ and a photoperiod of $12 \mathrm{~L}: 12 \mathrm{D}$ (similar protocol followed from Katandukila and Bennett 2016).

\section{Ethical note}

The research protocol was approved by the Animal Ethics Committee of the University of Pretoria (ethics clearance number ECO62-14), Pretoria, Republic of South Africa. Research clearances were granted by the Tanzania Wildlife Research Institute (TAWIRI) and Commission of Science and Technology (COSTECH: 2014-277-NA-2014-204). Heliophobius were maintained under the guidelines of the American Society of Mammalogists (Sikes et al. 2011; available from http://www.mammalsociety.org/articles/guidelines-american-society-mammalogists-use-wild- 
mammals-research). Export permits were authorized by TAWIRI, and granted by the Ministry of Natural Resources and Tourism, Wildlife Division (permit no. 68368) and animal export health permit no. 003986 from the Ministry of Livestock and Fisheries, Zoosanitary Section; in Dar es Salaam, Tanzania. The import permits (CPB6-2386 and 13/1/1/30/201411000171) were granted by the Gauteng Department of Nature Conservation and the Department of Agriculture, Forestry and Fisheries, Johannesburg, Republic of South Africa.

\section{Experimental design}

The experiment comprised a sequential design using the same six females $(172.83 \pm 31.59 \mathrm{~g})$ for all three protocols and the males $(175.8 \pm 34.73 \mathrm{~g})$ for the non control protocols. The particular design has been tried and tested in several other mole-rat species (Faulkes et al. 2010; Bennett et al. 2010; Katandukila et al. 2016) Experimental mole-rats were maintained under three consecutive experimental protocols namely, control (C), non-physical contact (NPC) and physical contact (PC). Each experimental treatment took place over 44 consecutive days; collection started in December 2015 and ended in April 2016. If the females exhibit spontaneous ovulation then they should exhibit ovarian cyclicity over all three protocols. In contrast, if they are induced ovulators then they should only exhibit ovarian cyclicity during the physical contact protocol.

Control (C). Six females were housed singly in cages $(34 \mathrm{~cm} \times 34 \mathrm{~cm} \times 20 \mathrm{~cm})$ with no males present in the same room, thus preventing any physical as well as chemical interaction between males and females. The rooms were individually ventilated and had a distinct inlet and exit ventilation. The protocol was used as a measure for baseline progesterone concentrations.

Non physical contact (NPC). Six females were housed in individual cages $(48 \mathrm{~cm}$ x $28.5 \mathrm{~cm} \mathrm{x}$ $48 \mathrm{~cm}$ ) and were exposed to one of the 5 individual males over the period of collection. A wire mesh separated the male from the female and thus prevented any physical contact. There was, 
however, visual, olfactory and auditory contact between the sexes. The males and females remained in these conditions for the duration of the experiment. The males were present for the entire 44 day period.

Physical contact (PC). Six females were paired with vasectomised males for a period of 6 hours every second day in cages $(48 \mathrm{~cm} \times 28.5 \mathrm{~cm} \times 48 \mathrm{~cm})$. This allowed for full physical contact as well as chemical contact of the females with the males and consequently the opportunity for copulations to occur. The daily 6 hour contact period occurred under supervision from the authors to ensure that no aggressive interaction resulted.

\section{Urine collection}

Urine was collected from female mole-rats $(n=6)$ on every second day at the onset of each experimental phase and labelled accordingly. Each female was kept in a cylindrical plastic cage with a wire mesh base settled on top of the urine collection tray; the wire mesh base prevented faecal material from entering the urine. Urine was collected between the hours of $08 \mathrm{~h} 00$ and $14 \mathrm{~h} 00$ so as to avoid any diel secretory patterns of progesterone secretion; as soon as urine had been voided, the female was placed back into the housing container. Urine was collected from December 2015 to April 2016; urine was kept in a freezer at $-40^{\circ} \mathrm{C}$ immediately following collection.

\section{Male vasectomy}

A qualified veterinarian vasectomized five male mole-rats 3 months prior to being placed in PC. Vasectomy was performed to remove the vas deferens and epididymis from each testis to ensure that no pregnancy resulted during the PC experimental phase. During vasectomy, the males were given mask-induced anaesthesia using 5\% isofluorane gas and subsequently maintained using $2 \%-2.5 \%$ isofluorane gas until the end of procedure. Meloxicam $(0.5 \mathrm{mg} / \mathrm{kg})$ was used as analgesia post surgery. Vasectomy was performed 3 months prior to experimentation to ensure the epididymis was clear of spematozoa 


\section{Assessment of penis morphology}

Two additional males were sacrificed in order to investigate the penile structure of Heliophobius mole-rats from Tanzania. This fixing procedure was followed by a buffer rinse in a $0.15 \mathrm{~mol} / \mathrm{L}$ phosphate buffer $(\mathrm{pH} 7.4)$. Penises were subjected to a series of dehydration stages using ethanol of increasing concentration (50-100\%). Critical point drying (CPD) was applied with liquid $\mathrm{CO}_{2}$ in a BIORAD 3000 critical point drier (Quorum Technologies, Watford, UK). The samples were mounted on double-sided carbon tape and then coated with carbon evaporation in an Emitek K950X (Quorum Technologies, Watford, UK). Samples were viewed with a scanning electron microscope (SEM model no. JSM-840: JEOL, Tokyo, Japan).

\section{Determination of creatinine}

Prior to the measurement of progesterone, the urine samples were analysed for creatinine concentration (Bonney et al. 1982) using a modified Jaffe reaction (Folin 1914).Standard curves $\left(R^{2}>0.99\right)$ were used to determine values of all samples. Urinary progesterone concentrations were expressed as nanogram per milligram creatinine (ng/mg $\mathrm{Cr}$ ); expressing urinary hormone concentrations relative to creatinine standardizes measurements over differing concentrations of urine.

\section{Radioimmunoassay}

Progesterone: A volume of $50 \mu \mathrm{L}$ of urine was dispensed in duplicate into polypropylene tubes following the Coat-A-Count progesterone kit (IBL International GMBH Germany) procedures. The Coat-A-Count progesterone antiserum is highly specific for progesterone with a low cross-reactivity $(<1 \%)$ to other naturally occurring steroids except in 5a-Pregnan- 
3, 20-dione (3.46\%), 20- $\beta$-Dihydroxyprogesterone (3.27\%) and 17- $\alpha$ - hydroxyprogesterone $(1.50 \%)$. The urine sample with a high concentration of progesterone was double-diluted using the assay buffer as a matrix $1: 1$ to $1: 32$ then assayed. The slopes of serial double dilution and standard curve were compared to check for parallelism (Analysis of covariance (ANCOVA): $\left.\left(F_{1,5}\right)=1.76 ; P>0.05 ; n=6\right)$ following a log-logit transformation of the data (Chard 1987). The intra-assay coefficient of variation for the urine pool was $5.8 \%$ whereas the inter-assay coefficient was $6.1 \%$ and sensitivity of the assay was $1.35 \mathrm{nmol} / \mathrm{L}$.

\section{Data analysis}

Data were analysed using $\mathrm{R}$ algorithms ( $\mathrm{R}$ Core team 2015). The data were tested for normality using ShapiroWilk Normality test $(W=0.852 P<0.05, n=396)$. A comparison of urinary progesterone between experimental phases/treatments and between females was undertaken using the Friedman statistical test for non-parametric parameters for related samples (Katandukila and Bennett 2016). The Wilcoxon Signed-rank statistical test was performed post hoc. Other measurements were expressed as mean \pm standard deviation (SD). Graphics were generated by Grammer of Graphics plotting2 (ggplot2) package in R programme. The level of significance for all statistical test was $\alpha \leq 0.05$. Due to variation of concentrations of progesterone between females in all experimental phases, a progesterone concentration of $>21 \mathrm{ng} / \mathrm{mg} \mathrm{Cr}$ was used as a cut-off point to signify the onset of luteal phase (i.e. luteal phase $=$ oestrus phase which indicates that ovulation has occured) as suggested in similar studies of bathyergids (Faulkes et al. 2010). 


\section{RESULTS}

\section{Cyclicity of ovarian hormone}

The mean concentration of urinary progesterone during the $(\mathrm{C})$ phase was $4.9 \pm 1.4 \mathrm{ng} / \mathrm{mg} \mathrm{Cr}$. In the (NPC) and the (PC) phases the mean concentrations of urinary progesterone were 10.7 $\pm 2.5 \mathrm{ng} / \mathrm{mg} \mathrm{Cr}$ and $22.7 \pm 8.9 \mathrm{ng} / \mathrm{mg} \mathrm{Cr}$, respectively.

The concentrations of urinary progesterone varied significantly between experimental phases

(Friedman's chi-square $=262.02, \mathrm{df}=2, P=0.0001<0.05, n=132$ ). The multiple comparisons of concentration of urinary progesterone between the three phases showed significantly higher concentrations of urinary progesterone during the PC phase than during either the $\mathrm{C}$ or NPC phases (C and NPC: $Z=-3.05, P=0.0023<0.05, n=132$; $\mathrm{C}$ and PC: 9.97, $P=0.0001<0.05, n=132 ; \mathrm{NPC}$ and $\mathrm{PC}: Z=-6.46, P=0.0001<0.05, n=132)$.

The mean concentration of urinary progesterone varied significantly between the three experimental protocols in all females, with slight variation between females (Table 1). Three individuals had higher concentrations of progesterone, although both groups of females showed the same general trend in their progesterone profiles (Figure 1). Variation in the concentration of urinary progesterone between females was not statistically significant (Friedman's $\chi^{2}=8.25, \mathrm{df}=5, P=0.143>0.05, n=66$ ). Copulation arose on the first day of PC in all six female Heliophobius (see Figure 1). A follicular phase of $10 \pm 1.8$ days was estimated, whereas the elevation of urinary progesterone concentrations had a mean (SD) length of $20.3 \pm 0.8$ days, and.

\section{Penile morphology}


The SEM revealed numerous spines on the outer surface of the glans of the two penises (Figure 2A). These pointed projections were unevenly distributed, at higher magnification the protruding spines overlapped the skin.

\section{DISCUSSION}

The sequential experiments investigating the pattern of ovulation in captive female Heliophobius confirmed that the species is an induced ovulator. The highest concentration of urinary progesterone in captive females demonstrated that ovulation took place during physical contact when paired with an unrelated conspecific male. Female mole-rats showed raised concentrations of progesterone a few days after the initial recordings of copulation indicative of an initial follicular and a subsequent luteal phase. The tactile stimuli resulting from the outer surface of penis during copulation against the vaginal wall and cervix has been reported to be important in bringing on ovulation for induced ovulators (Jöchle 1975; Viker et al. 1993; Parag et al. 2006).

The elevation of urinary progesterone concentrations had a mean (SD) length of $20.3 \pm 0.8$ days, and prior to the proposed luteal phase, a follicular phase of $10 \pm 1.8$ days is estimated. The available data suggests a length of the ovarian cycle of approximately one month (30 days). The ovarian cycle is thus longer than that of the 26 days derived for the giant mole-rat, Fukomys mechowii (Faulkes et al. 2010) or the 27.5 days for the naked mole-rat (Faulkes et al. 1990). Since the females used for this experiment had baseline concentrations at the start of the experiment, it was assumed that these females were acyclic prior to the start of the experiments.

The profile of the urinary progesterone in captive female Heliophobius coincides with the general trend reported in previous studies of subterranean rodents including the Cape dune 
mole-rat (Batherygus suillus; Bennett et al. 2000), social Natal mole-rat (Cryptomys hottentotus natalensis; Jackson and Bennett 2005), social highveld mole-rat (Cryptomys hottentotus pretoriae; Malherbe et al. 2005) and Cape mole-rat (Georychus capensis; van Sandywk and Bennett 2005).

In the NPC situation the slight rise in the progesterone concentrations highlights that the physical presence but no contact may trigger a rise in progesterone. The rise in progesterone may arise from the luteinisation of unrupted follicles that fail to ovulate because of a lack of coitus. Luteinised unruptured follicles have been found to be a source of progesterone in non cycling females (Bennett et al. 1994).

The onset of significant elevation of progesterone hormone which signified the luteal phase arose after copulation. The first copulation in the species is shorter in duration than that reported in other subterranean bathyergids (Faulkes et al. 1990, 2010). Katandukila and Bennett (2016) intimate that semio-chemical and seismic communication during non-physical contact plays an important role in relaxing agonistic activity. Seismic signalling occurs with the different sexes announcing their presence and sexual status by displaying a particular frequency of foot/tooth drumming, (Bennett and Jarvis 1988; Narins et al. 1992; Bennett et al. 1999; Katandukila and Bennett 2016). Both sexes of were recorded to display teeth rapping when separated by wire mesh, but when housed together the agonistic behaviour disappeared. This observation supports previous studies on solitary rodents demonstrating that courtship and subsequent copulation is a brief affair and one that requires the highly aggressive and xenophobic behaviours that are characteristic of these species to be relaxed (Bennett and Jarvis 1988; Narins et al. 1992; Bennett et al. 2000; Fanjul and Zenuto 2012; Katandukila and Bennett 2016). 
Heliophobius a. Emini breeds seasonally with a clear single breeding cycle of one litter per annum (Šumbera et al. 2003a; Ngalameno et al. 2017). Heliophobius a. emini They exhibit lower concentrations of steroid hormone and reduced gonadal size from September through to early May with pups born from the end of August to October, signifying that mating occurs towards the end of May (Šumbera 2003a; Ngalameno et al. 2017). In Kenya, the silvery molerat is reported to have pups from April to July with mating speculated to occur from January to March (Copley 1950), whereas in Zambia and Malawi mating reportedly starts at the end of May and June with pups born in August (Scharff et al. 2001; Šumbera et al. 2003a). These latter studies suggest that onset of breeding season in silvery mole-rats occurs towards the end of the rains (Jarvis 1969; Sumbera et al. 2003a), perhaps as a consequences of the long gestation period. Noteworthy is that in Tanzania, pups were born during the short rainy season (September to October) with sub-adults dispersing in December when they extend their burrows to secure more geophytes. Rainfall is of great importance to subterranean rodents including Heliophobius as it facilitates the growth of geophytes for energy acquisiton and workable soil for dispersal of sub-adults (Bennett and Faulkes 2000).

The penile morphology of male Heliophobius exhibits similar patterns to the males of other solitary species possessing epidemal protrusions on the glans. Penile spines stimulate the cervix during coitus and subsequently the release of gonadotrophin releasing hormone (GnRH) from the hypothalamus and subsequent cascade of hormones responsible for ovulation (Milligan 1980; Sellix and Menaker 2010). Penile spines are found in other species of the family Bathyergidae, including B. suillus and G. capensis (Parag et al. 2006). Other subterranean rodents possessing penile spines include the blind mole-rat, Spalax ehrenbergi (Shanas et al. 1995), the tuco tuco, C. talarum (Fanjul and Zenuto 2012) and the East African root rat, T. splendens, (Katandukila and Bennett 2016). The social bathergids reported to exhibit induced ovulation ( $C$. hottentotus natalensis and $C$. hottentotus pretoriae; Malherbe et 
al. 2004) have small protrusions at the apex of their glans, an ornamentation that may play the same role as penile spines (Malherbe et al. 2004; Jackson et al. 2005; Parag et al. 2006).

Comparison of the shape of spines on the outer surface of penis between solitary subterranean rodents showed that Heliophobius a emini had spines of similar shape to the Cape dune molerat, Bathyergus suillus (Parag et al. 2006). These two species both have longer and sharper pointed spines than those of the Cape mole-rat, Georychus capensis and the East African rootrat, Tachyoryctes splendens which both exhibit shorter spines (Figure 2B) (Van Sandwyk and Bennett 2005; Katandukila and Bennett 2016).

Currently all solitary and social species of bathyergid studied so far that exhibit a seasonality to reproduction also show induced pattern have males with epidermal spines on the glans penis and shaft (Parag et al. 2006). In contrast, in the social species of the genus Heterocephalus and Fukomys, the penis lacks ornamentation and is smooth or grooved (Parag et al. 2006; Bennett et al. 2010; Faulkes et al. 2010) and the progesterone profiles in these species exhibit luteal peaks when housed singly, in non physical contact or with the presence of a vasectomized male. These findings emphasize the sexual selective forces shaping evolution in response to the degree of sociality and morphological ornamentation on the penis. Faulkes et al. (2004), proposed a phylogeographic scenario for the current distribution of many species of African mole-rat. The study suggested that the 'primordial' bathyergid was East African and that with subsequent migration and speciation of this progenitor a northsouth migration of bathyergids arose that ended subsequently in central Africa. Interestingly, many of the southern African species exhibiting a seasonal pattern of reproduction are either solitary or loosely social. These have been reported to exhibit an induced pattern of ovulation. The assumption is that ancestral bathyergids exhibited spontaneous type of ovulation, but as they migrated and inhabited seasonal habitats and with complete lose of their social behaviour 
(Georychus, Bathyergus and Heliophobius) or some degree of sociality (Cryptomys), they adopted an induced type of ovulation in order to reproduce rapidly.

Induced ovulation in small mammals is crucial because most of them have short life spans and as a consequence time for perpetuation of their genes to the next generation is limited. By exhibiting an induced pattern of ovulation the chance for breeding increases and also prevents the waste of unfertilized egg production in females. This helps to conserve energy which is crucial to their short lifespans.

Heliophobius is a strictly solitary species where the different sexes come together to breed during the period of the long rains in East Africa. Females when they pair with a male and copulate exhibit raised levels of progesterone due to the mechanical stimulation provided by the epidermal spines of the ornamented penis of the male. This study confirms that Heliophobius a. emini is an induced ovulator and this fits in with the general trend exhibited within the family Bathyergidae (Parag et al. 2006).

\section{Table legends}

Table 1. The summary of concentration progesterone (ng/mg Cr: mean $\pm \mathrm{SD}$ ) from experimental female silvery mole-rats $(\mathrm{F} 1-\mathrm{F} 6 ; n=6)$ within three experimental phases $(\mathrm{C}=$ control; NPC $=$ non-physical contact $; \mathrm{PC}=$ physical contact $)$.

\section{Figure legends}

Figure 1. Trend of concentration of urinary progesterone of six female silvery mole-rats (Heliophobius argenteocinereus emini) with respective dates (day/month/year) of urine collection during the three experimental phases $\mathrm{C}=$ control, $\mathrm{NPC}=$ non physical contact, $\mathrm{PC}=$ physical contact, $\mathrm{F}=$ female, $\mathrm{Cr}=$ creatinine. Arrows indicate days when copulation was observed, progesterone values are for evert second day of collection. 
Figure 2A: Surface morphology of the penis of male Heliophobius argenteocinereus seen with Scanning Electronic Microscope (SEM); i = outer suraface of the penis, $i i=$ magnified protuding structure on the penile surface: $\mathrm{A}=$ protuding structures like spines, $\mathrm{B}=$ magnified spine-like structure of the penis.

Figure 2B: Morphology of outer surface on penile structure of solitary African subteraneanrodents; $\boldsymbol{a}=$ Bathyergus suillus; $\boldsymbol{b}=$ Georychus capensis $; \boldsymbol{c}=$ Heliophobius argenteocinereus; $\boldsymbol{d}=$ Tachyoryctes splendens (source for $\boldsymbol{a}$ and b: Parag et al. 2006; c: Katandukila et al. 2016).

Note: Bathyergus suillus, Georychus capensis and Heliophobius argenteocinereus belong to the family bathyergidae while Tachyoryctes splendens belongs to the family spalacidae (see introduction for details).

\section{Acknowledgements}

We gratefully acknowledge financial support from the University of Pretoria (International UP Funds), South Africa. We thank the Tanzania Wildlife Research Institute (TAWIRI) and Tanzania Commission for Science and Technology (COSTECH) for granting capture permit and authorising the export permits. We also thank Ministry of Wildlife and Tourism (Wildlife Division) and Ministry of Livestock and Fisheries (Zoosanitary Section) all of Tanzania for granting export permits. We also gratefully acknowledge financial support from the South African Research Chair of Mammal Behavioural Ecology and Physiology from the DST-NRF to NCB. We also acknowledge Alan Hall and Erna van Wilpe of the Microscopy section, University of Pretoria for their assistance and expertise. 


\section{References}

Bennett, N. C., and Jarvis, J. U. M. 1988. The reproductive biology of the Cape mole-rat, Georychus capensis (Rodentia, Bathyergidae). J. Zool. (Lond.) 214: 95-106.

Bennett, N.C., Jarvis, J.U.M., Millar, R.P., Sasano, H., and Ntshinga, K.V. 1994. Reproductive suppression in eusocial Cryptomys damarensis: Colonies social-induced infertility in females. J. Zool. (Lond.) 233: 617-630.

Bennett, N.C. and Faulkes, C.G. 2000. African Mole-rats: Ecology and Eusociality. Cambridge University Press, Cambridge, U.K.

Bennett, N.C., Faulkes, C.G., and Molteno, A.J. 2000. Reproduction in subterranean rodents. In Life underground, the biology of subterranean rodents. Edited by G.N. Cameron, E.A. Lacey and J. Patton. Chicago University Press, Chicago pp. 145-177

Bennett, N.C., Lutermann, H., and van Sandwyk, J.H. 2010. The pattern of ovulation in Ansell's molerat, Fukomys anselli: phylogenetic or ecological constraints? J. Zool. (Lond.). 281: 66-73.

Bonney, R.C., Wood, D.J., and Kleimant, D.G. 1982. Endocrine correlates of behavioural oestrus in the female giant panda (Ailuropoda melanoleuca) and associated hormonal changes in the male. J. Reprod. Fertil. 64: 209-215.

Chard, T. 1987. An introduction to radioimmunoassay and related techniques. Amsterdam Elsevier, The Netherlands.

Conaway, C.H. 1971. Biological adaptation and mammalian reproduction. Biol. Reprod. 4: 239-247.

Copley, H. 1950. Small mammals of Kenya. Highway Press, Nairobi, Kenya.

Fanjul, M.S., and Zenuto, R.R. 2012. Female reproductive behaviour, ovarian hormones and vaginal cytology of the induced ovulator, Ctenomys talarum. Acta Theriol. 57: 15-27.

Faulkes, C.G., Abbott, D.H., and Jarvis, J.U. M. 1990. Social suppression of ovarian cyclicity in captive and wild colonies of naked mole-rats, Heterocephalus glaber. J. Reprod. Fertil. 88: 559-568.

Faulkes, C.G., Verheyen, E., Verheyen, W. Jarvis, J.U.M., and Bennett, N.C. 2004. Phylogeography and speciation in African mole-rats (Family: Bathyergidae). Mol. Ecol. 13: 613-629.

Faulkes, C.G., Sichilima, A.M., Van Sandwyk, J., Lutermann, H., and Bennett, N.C. 2010. Control of ovulation in female giant mole-rats Fukomys mechowii (Rodentia: Bathyergidae), and phylogenetic trends within the family. J. Zool. (Lond.). 282: 64-74.

Faulkes, C.G., Bennett, N.C., Cotterill, F.P.D., Stanley W., Mgode, G.F., and Verheyen, E. 2011. Phylogeography and cryptic diversity of the solitary-dwelling silvery mole-rat, genus Heliophophobius (family: Bathyergidae). J. Zool. (Lond.). 285: 324-338.

Feder, H.H. 1981. Estrous cyclicity in mammals. In Neuroendocrinology of reproduction. Edited by N.T. Adler. Plennum Publishers, USA pp. 279-334.

Folin, O. 1914. On the determination of creatinine and creatinine the urine. J. Biol. Chem. 17: 469473.

Hart, L., O'Riain, M.J., Jarvis, J.U.M., and Bennett, N.C. 2006. Is the Cape Dune Mole-Rat, Bathyergus suillus (Rodentia: Bathyergidae), a Seasonal or Aseasonal Breeder? J. Mammal. 87: 1078-1085.

Herbst, M., Jarvis, J.U.M., and Bennett, N.C. 2004. A field assessment of reproductive seasonality in the threatened wild Namaqua dune mole-rat (Bathyergus janetta). J. Zool. (Lond).263: 259268.

Herbst, M., and Bennett, N.C. 2006. The burrow architecture and burrowing dynamics of the endangered Namaqua dune mole-rat, Bathyergus janetta (Rodentia: Bathyergidae). J. Zool. (Lond.). 270: 420-428. 
Heth, G. 1989. Burrow patterns of the mole-rat Spalax ehrenbergi in two soil types (terra-rossa and rendzina) in Mount Carmel, Israel. J. Zool.(Lond.). 217: 39-56.

Hickok, J.R., and Tischkau, S.A. 2010. In vivo circadian rhythms in gonadotropin-releasing hormone neurons. Neuroendocrinology, 91: 110-120.

Jackson, C.R. and Bennett, N.C. 2005. Is the Natal mole-rat (Cryptomys hottentotus natalensis) a spontaneous or induced ovulator?. J Mammal. 86: 1-6.

Jarvis, J.U.M. 1969. Some aspects of the biology of East African mole-rats. PhD thesis. University of East Africa, Nairobi, Kenya.

Jarvis, J.U.M., and Sale, J.B. 1971. Burrowing and burrow structure of East African mole-rats Tachyoryctes, Heliophobius and Heterocephalus. J. Zool. (Lond.). 163: 451-479.

Jöchle, W. 1975. Current research in coitus-induced ovulation: a review. J. Reprod. Fertil. Supplement, 22:165-207.

Katandukila, J.V., Chimimba, C.T., Bennett, N.C., Makundi, R.H., Le Comber, S.C., and Faulkes, C.G. 2014. Sweeping the house clean: burrow architecture and seasonal digging activity in the East African root rat from Tanzania. J. Zool. (Lond.). 293: 271-280.

Katandukila, J.V., and Bennett, N.C. 2016. Pattern of ovulation in the East African root rat (Tachyoryctes splendens) from Tanzania: induced or spontaneous ovulator? Can. J. Zool. 94: $345-351$.

Lariviere, S., and Ferguson, S.H. 2003. Evolution of induced ovulation in North American carnivores. J.Mammal. 84: 937-947.

Malherbe, G.P., Bennett, N.C., and Schoeman, A.S. 2004. Is the highveld mole-rat Cryptomys hottentotus pretoriae (Rodentia: Bathyergidae) an induced or spontaneous ovulator? J. Zool. (Lond.). 263: 159-165.

Milligan, S.R. 1980. Effect of bromocryptine treatment on the ovulatory response to oestradiol benzoate in the reflex ovulator, Microtus agrestris. J Endocrinol. 84: 315-316.

Muteka, S.P., Chimimba, C.T and Bennett, N.C. 2006. Reproductive seasonality in Aethomys namaquensis (Rodentia: Muridae) from southern Africa. J. Mammal. 87: 67-74.

Narins, P. M., Reichman, O., Jarvis, J. U.M., and Lewis, E. R. 1992. Seismic signal transmission between burrows of the Cape mole-rat, Georychus capensis. J. Comp. Physiol. A: Neur. Sens. Neur.Behav. Physiol. 170: 13-21.

Ngalameno, M.K., Bastos, A.D.S., Mgode, G.F., and Bennett, N.C. 2017. The pattern of reproduction Heliophobius from Tanzania: do not refrain during the long rains! Can J. Zool. 95: 107-114.

Parag, A., Bennett, N. C., Faulkes, C. G., and Bateman, P. W. 2006. Penile morphology of African mole-rats (Bathyergidae): structural modification in relation to mode of ovulation and degree of sociality. J. Zool. (Lond.). 270: 323-329.

R Core Team. 2015. R: A language and environment for statistical computing. R Foundation for Statistical Computing, Vienna, Austria. Available from http://www.R-project.org/.

Scharff, A., Macholan, M., Zima, J., and Burda, H. 2001. A new karyotype of Heliophobius argenteocinereus (Bathyergidae, Rodentia) from Zambia with field notes on the species. Mamm. Biol. 66: 376-378.

Sellix, M.T. and Menaker, M. 2010. Circadian clocks in the ovary. Trends Endocrinol. Metab. 21: 628-636.

Shanas, U., Heth, G., Nevo, E., Shalgi, R. and Terkel, J. 1995. Reproductive behaviour in the female blind mole (Spalax ehrenbergi). J. Zool. (Lond.) 237: 195-210.

Sikes, R.S., Gannon, W.L., and Animal Care and Use Committee of the American Society of Mammalogists. 2011. Guidelines of the American Society of Mammalogists for the use of wild mammals in research. J. Mammal. 92: 235-253. 
Šklíba, J., Šumbera, R., Chitaukali, W., and Burda, H. 2007. Determinants of daily activity patterns in a free-living Afrotropical solitary subterranean rodent. J. Mammal. 88(4): 1009-1016.

Snyman, P.C., Jackson, C.R., and Bennett, N.C. 2006. Do dispersing non-reproductive female Damaraland mole-rats, Cryptomys damarensis (Rodentia: Bathyergidae) exhibit spontaneous or induced ovulation?. Physiol. Behav. 87: 88-94.

Šumbera, R., Burda, H., and Chitaukali, W.N. 2003a. Reproductive biology of a solitary subterranean bathyergid rodent, The Silvery mole-rat (Heliophobius argenteocinereus). J. Mammal. 84: 278-287.

Šumbera, R., Burda, H., Chitaukali, N. W., and Kubová, J. 2003b. "Silvery mole-rats (Heliophobius argenteocinereus, Bathyergidae) change their burrow architecture seasonally." Naturwissenschaften, 90: 370-373.

Thomas, H.G., Bateman, P.W., Le Comber, S.C., Bennett, N.C., Elwood, R.W., and Scantlebury, M., 2009. Burrow architecture and digging activity in the Cape dune mole-rat. J. Zool. (Lond.). 279: $277-284$.

Van Sandwyk, J.H.D.T., and Bennett, N.C. 2005. Do solitary, seismic signalling Cape mole-rats (Georychus capensis) demonstrate spontaneous or induced ovulation?. J. Zool. (Lond.). 267: 75-80.

Viker, S.D., Larson, R.L., Kiracofe, G.H., Stewart, R.E., and Stevenson, J.S. 1993. Prolonged postpartum anovulation in mastectomized cows requires tactile stimulation by the calf. J. Anim. Sci. 71: 999-1003. 

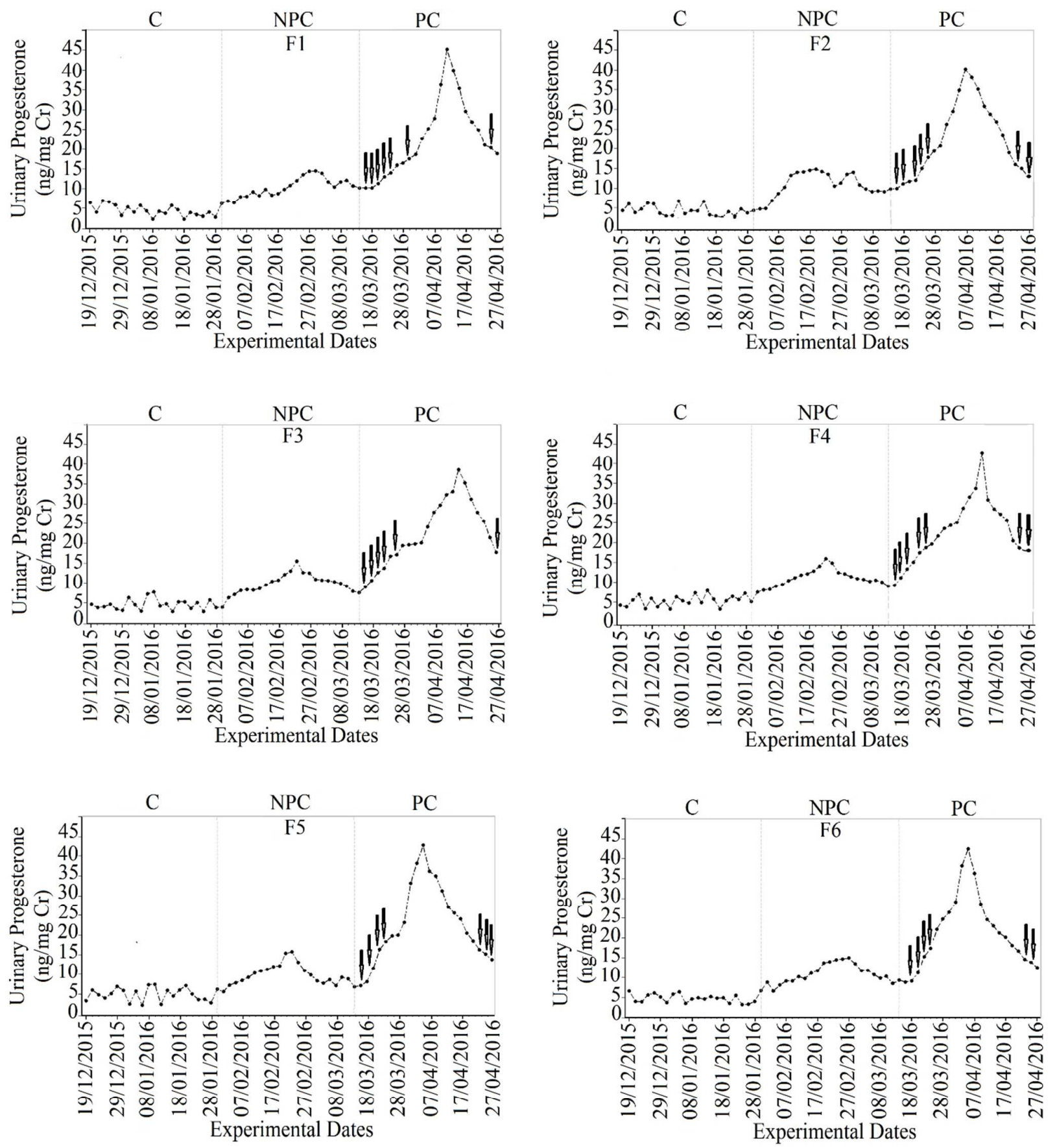

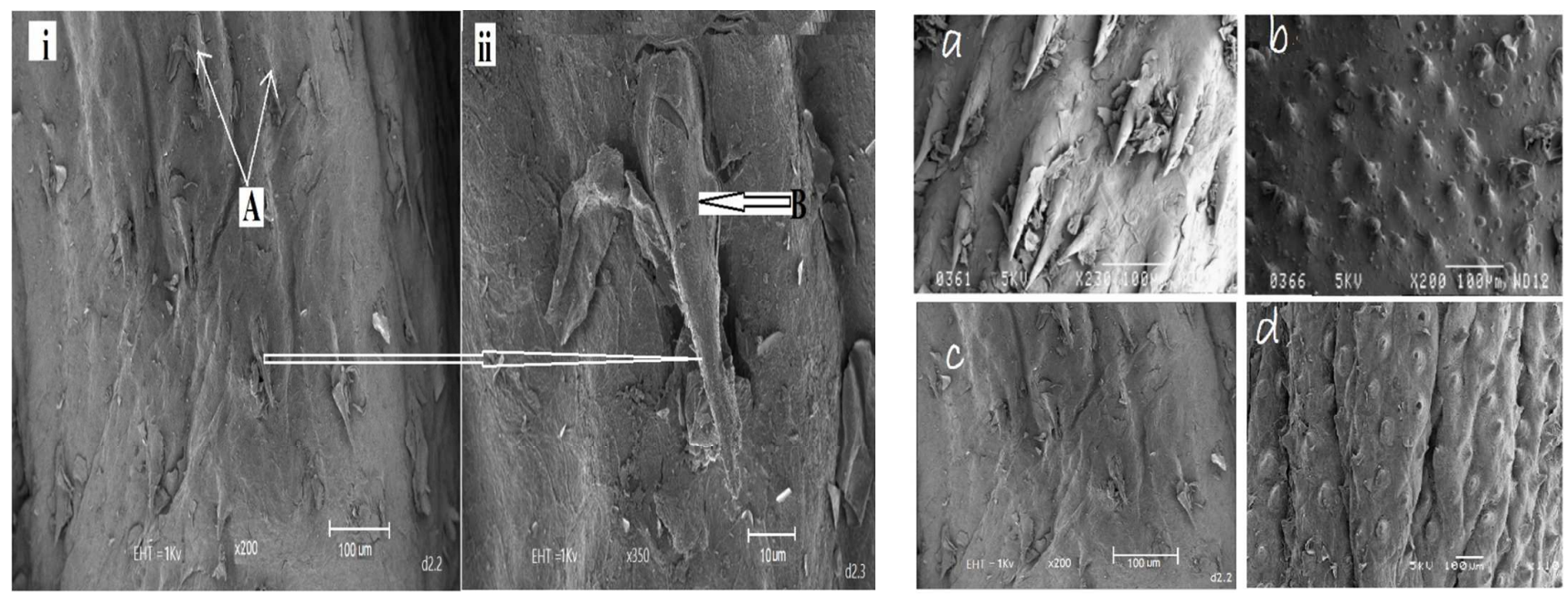

$2 \mathrm{~A}$

2B

https://mc06.manuscriptcentral.com/cjz-pubs 
Table 1: The summary of concentration progesterone (ng/mg Cr: mean \pm SD) from experimental female silvery mole-rats $(\mathrm{F} 1-\mathrm{F} 6 ; n=6)$ within three experimental phases $(\mathrm{C}=$ control; NPC $=$ non-physical contact; $\mathrm{PC}=$ physical contact $)$.

\begin{tabular}{|l|l|l|l|l|}
\hline \multirow{2}{*}{ Individuals } & \multicolumn{4}{|l}{ Concentration of urinary progesterone (ng/mg Cr) } \\
\cline { 2 - 5 } & overall & C & NPC & PC \\
\hline F1 & $12.57 \pm 9.56$ & $4.55 \pm 1.40$ & $10.43 \pm 2.35$ & $22.73 \pm 9.73$ \\
\hline F2 & $12.65 \pm 9.47$ & $4.34 \pm 1.29$ & $11.04 \pm 3.07$ & $22.57 \pm 9.40$ \\
\hline F3 & $12.44 \pm 9.20$ & $4.47 \pm 1.34$ & $9.99 \pm 2.24$ & $22.87 \pm 8.27$ \\
\hline F4 & $13.08 \pm 8.80$ & $5.29 \pm 1.35$ & $11.01 \pm 2.16$ & $22.95 \pm 8.24$ \\
\hline F5 & $12.68 \pm 9.60$ & $5.09 \pm 1.67$ & $9.99 \pm 2.65$ & $22.95 \pm 9.85$ \\
\hline F6 & $13.08 \pm 8.91$ & $5.39 \pm 1.10$ & $11.60 \pm 2.39$ & $22.25 \pm 8.31$ \\
\hline
\end{tabular}

$\mathrm{Cr}=$ creatinine, $\mathrm{F}=$ female

Note: The concentration of urinary progesterone between experimental phases varied significantly in all females, but variation in concentrations between females was insignificant (see results above for details). 